\title{
Hidroquímica y geoacumulación de metales en sedimentos superficiales de la Laguna de Metztitlán, Hgo., México
}

\author{
Hydrochemistry and geoaccumulation of metals in superficial sediments of the Laguna de \\ Metztitlán, Hgo., México \\ Misael Cruz-Sánchez ${ }^{a}$, Anallely Avila-Ortíz ${ }^{a}$, Violeta Lobaco-Mejía ${ }^{a}$, Patricia Girón-García ${ }^{b}$, \\ Cecilia Salcedo-Luna ${ }^{c}$
}

a Área Académica de Ciencias de la Tierra y Materiales, Universidad Autónoma del Estado de Hidalgo, Mineral de la Reforma, Hidalgo, 42184, México.

${ }^{\mathrm{b}}$ Departamento de Geoquímica, Instituto de Geología, Universidad Nacional Autónoma de México, Circuito de la Investigación Científica S/N, Alcaldía de Coyoacán, Ciudad de México, 04510, México.

c USAI, Facultad de Química, Universidad Nacional Autónoma de México, Circuito de la Investigación Científica S/N, Alcaldía de Coyoacán, Ciudad de México, 04510, México.

\begin{abstract}
:
The Metztitlán lagoon is a shallow natural reservoir that is part of the Barranca de Metztitlán Biosphere Reserve; it is a highly vulnerable system due to the incorporation of wastewater, as well as seasonal variations in rain-drought cycles. The physicochemical evaluation of a series of water samples and superficial sediments allowed classifying the nature of the water as lithotrophic with a Ca-Mg-HCO 3 facie, C2S1 salinity, and an average permeability of $44.53 \%$ that make it suitable for irrigation. Sediment mineralogy is mainly defined by calcite, quartz, plagioclases, and kaolinite. The evaluation of the enrichment factor and the geoaccumulation index showed a moderate enrichment of $\mathrm{V}, \mathrm{Mn}, \mathrm{Cu}, \mathrm{Zn}$, and $\mathrm{Pb}$ in the sediments, in addition to a positive geoaccumulation for $\mathrm{V}, \mathrm{Zn}$ and $\mathrm{Pb}$, indicating possible contamination with these metals.
\end{abstract}

Keywords:

Water, facie, sediments, minerals, metals

\section{Resumen:}

La laguna de Metztitlán es un embalse natural somero que forma parte de la Reserva de la Biosfera Barranca de Metztitlán; es un sistema altamente vulnerable debido a la incorporación de aguas residuales, así como por las variaciones estacionales en los ciclos de lluvia-sequía. La evaluación fisicoquímica de una serie de muestras de agua y sedimentos superficiales permitió clasificar la naturaleza del agua como litotrófica con una facie Ca-Mg- $\mathrm{HCO}_{3}$, salinidad $\mathrm{C} 2 \mathrm{~S} 1$ y una permeabilidad promedio de $44.53 \%$ que la hacen apta para el riego. La mineralogía de los sedimentos está definida principalmente por calcita, cuarzo, plagioclasas y caolinita. La evaluación del factor de enriquecimiento y el índice de geoacumulación demostraron un enriquecimiento moderado de $\mathrm{V}, \mathrm{Mn}, \mathrm{Cu}, \mathrm{Zn}$ y $\mathrm{Pb}$ en los sedimentos, además de una geoacumulación positiva para $\mathrm{V}, \mathrm{Zn}$ y Pb, indicando una posible contaminación con estos metales.

\section{Palabras Clave:}

Agua, facie, sedimentos, minerales, metales

\section{Introducción}

La laguna de Metztitlán es un embalse natural somero localizado entre los municipios de Metztitlán y Eloxochitlán en el Estado de Hidalgo. Es uno de los humedales más importantes de la Reserva de la Biosfera Barranca de Metztitlán, en el área predomina el matorral xerófilo, vegetación ribereña y ocasionalmente el bosque caducifolio. A la laguna se asocian
198 especies de flora y 303 de fauna, algunas de las cuales se encuentran en riesgo [1].

El origen de la laguna está relacionado al deslizamiento de una ladera que generó un gran depósito de rocas que, a manera de represa, obstruyó el cauce del río Metztitlán en su trayecto hacia el río Almolón [2]. La consecuencia de este evento fue el desarrollo de la vega de Metztitlán, misma que desde tiempos

Autor para la correspondencia: mcruz@uaeh.edu.mx

Correos electrónicos: mcruz@uaeh.edu.mx (M. Cruz-Sánchez), anallely_avila10387@uaeh.edu.mx (A. Avila-Ortíz), lo297949@uaeh.edu.mx (V. Lobaco-Mejía), pgiron@geologia.unam.mx (P. Girón-García), cecy1269@yahoo.com.mx (C. Salcedo-Luna).

ORCID: https://orcid.org/0000-0001-8574-5690 (M. Cruz-Sánchez) 
prehispánicos fue utilizada para la siembra de diversos productos agrícolas como maíz, frijol, chile, calabaza, chía y algodón, principalmente [3].

Las variaciones en los ciclos de lluvia y sequía promueven el aumento y contracción de la laguna de manera periódica, modificando su volumen a lo largo del año, siendo los meses de julio a noviembre los periodos de inundación y de diciembre a junio los de estiaje [4]. A través de los años se han documentado periodos de inundación severa que han afectado notablemente la vega de Metztitlán, por ello, a principios del siglo XX se construyeron dos túneles para optimizar su desfogue hacia el río Almolón y reforzar el drenaje subterráneo que de manera natural había regulado el volumen de la laguna [5].

La laguna de Metztitlán juega un papel muy importante desde la perspectiva ecológica, regulando y amortiguando no solo el clima de la región, sino también el impacto de las aportaciones de sedimentos, materia orgánica, residuos domésticos, industriales y agrícolas que son transportados a través de las diferentes corrientes fluviales que convergen en ella.

Cuando una partícula, ión o molécula ingresa a la laguna, interactúa con un medio que está en constante movimiento debido a la presencia de fuertes vientos procedentes del Golfo de México, favoreciendo su mezcla e interacción química con los sedimentos, mismos que le confieren una elevada turbidez al cuerpo de agua, sobre todo si se considera que la profundidad de la laguna fluctúa entre los $11 \mathrm{~m}$ en su parte más profunda hasta sólo algunos centímetros en sus márgenes.

Los diferentes procesos fisicoquímicos tales como la adsorción, absorción, reacciones de óxido-reducción, ácido-base, complejación y precipitación pueden actuar de manera cooperativa para promover la depuración del medio acuático hasta una condición de equilibrio, en cuyo caso, tanto el agua como los sedimentos conservarán un registro de ésta interrelación.

El objetivo de la investigación fue evaluar las propiedades fisicoquímicas de una serie de muestras de agua y sedimentos superficiales periféricos, para determinar las características hidroquímicas de la laguna y establecer su interrelación con la acumulación de metales en los sedimentos de la misma.

\section{Descripción del área de estudio}

La laguna de Metztitlán se ubica a $15 \mathrm{Km}$ al norte de la cabecera municipal del mismo nombre, en el Estado de Hidalgo, México. Geográficamente se localiza entre las coordenadas $20^{\circ} 40^{\prime}$ y $20^{\circ} 42^{\prime}$ de latitud norte y entre $98^{\circ} 50^{\prime}$ y $98^{\circ} 53^{\prime}$ de longitud oeste. El clima dominante es BSOhw, seco semicálido con lluvias en verano. La precipitación media anual es de 400 a 450 $\mathrm{mm}$, con un porcentaje de precipitación invernal de 5 a $10 \%$ con invierno fresco [6].

La laguna se encuentra en la provincia de la Sierra Madre Oriental, dentro de la subprovincia del Carso Huasteco. Esta subprovincia es una zona de sierras plegadas constituidas predominantemente por calizas. El carso presenta un fuerte grado de disección por la acción de los ríos que fluyen en él [7]. En la zona de estudio afloran la caliza y la dolomía de la Formación El Abra (Albiano-Cenomaniano), a la cual le sobreyace una secuencia de lutita, arenisca, limolita y caliza arcillosa de las Formaciones Soyatal (Turoniano-Campaniano) y Méndez (Campaniano-Maastrichtiano). A estas formaciones sobreyace la Formación Terciaria Atotonilco El Grande, representada por derrames de andesita basáltica, toba andesítica, dacítica y riolítica, cubierta parcialmente por derrames andesítico-basálticos y conglomerado hacia la cima de la Formación Tarango (Plioceno-Pleistoceno). El aluvión corresponde al Cuaternario y está constituido por fragmentos de rocas preexistentes. La laguna de Metztitlán se localiza en la parte norte del Anticlinorio Huayacocotla, el cual está formado por pliegues y cabalgaduras de tipo chevrón originados durante la Orogenia Laramide ocurrida a finales del Cretácico Tardío y principios del Terciario [8].

La laguna de Metztitlán pertenece a la Región Hidrológica (RH26) Cuenca del Río Pánuco. El principal rasgo hidrológico superficial lo conforma un río que a lo largo de su recorrido cambia de nombre de acuerdo a la región; identificándose tres secciones importantes: la primera sección se localiza al sur de la Barranca de Metztitlán y se conoce como río Grande Tulancingo; la segunda, en la unión con el río San Agustín, se conoce como río Venados y la tercera, al norte de la Reserva, donde inicia el Distrito 08 Metztitlán, adopta el nombre de río Metztitlán que desemboca en la laguna [9].

\section{Desarrollo experimental}

\subsection{Descripción de las muestras}

En el mes de marzo de 2018 se colectaron 12 muestras de agua de la laguna de Metztitlán empleando frascos de polietileno con capacidad de $2 \mathrm{~L}$. Las muestras fueron etiquetadas y trasladadas en una hielera al laboratorio, conservándolas en refrigeración a $4{ }^{\circ} \mathrm{C}$ hasta el momento de su análisis, el cual no fue mayor a 72 h (tabla I, figura 1).

Tabla I. Coordenadas de toma de muestras de agua.

\begin{tabular}{|l|l|l|}
\hline Clave & Latitud (N) & Longitud (W) \\
\hline A1 & $20^{\circ} 41^{\prime} 55^{\prime \prime}$ & $98^{\circ} 52^{\prime} 17^{\prime \prime}$ \\
\hline A2 & $20^{\circ} 41^{\prime} 34^{\prime \prime}$ & $98^{\circ} 52^{\prime} 58^{\prime \prime}$ \\
\hline A3 & $20^{\circ} 41^{\prime} 32^{\prime \prime}$ & $98^{\circ} 52^{\prime} 10^{\prime \prime}$ \\
\hline A4 & $20^{\circ} 41^{\prime} 24^{\prime \prime}$ & $98^{\circ} 52^{\prime} 34^{\prime \prime}$ \\
\hline A5 & $20^{\circ} 41^{\prime} 01^{\prime \prime}$ & $98^{\circ} 52^{\prime} 18^{\prime \prime}$ \\
\hline A6 & $20^{\circ} 40^{\prime} 56^{\prime \prime}$ & $98^{\circ} 52^{\prime} 52^{\prime \prime}$ \\
\hline A7 & $20^{\circ} 40^{\prime} 44^{\prime \prime}$ & $98^{\circ} 51^{\prime} 54^{\prime \prime}$ \\
\hline A8 & $20^{\circ} 40^{\prime} 27^{\prime \prime}$ & $98^{\circ} 52^{\prime} 16^{\prime \prime}$ \\
\hline A9 & $20^{\circ} 40^{\prime} 02^{\prime \prime}$ & $98^{\circ} 52^{\prime} 06^{\prime \prime}$ \\
\hline A10 & $20^{\circ} 41^{\prime} 51^{\prime \prime}$ & $98^{\circ} 51^{\prime} 10^{\prime \prime}$ \\
\hline A11 & $20^{\circ} 41^{\prime} 22^{\prime \prime}$ & $98^{\circ} 51^{\prime} 15^{\prime \prime}$ \\
\hline A12 & $20^{\circ} 41^{\prime} 28^{\prime \prime}$ & $98^{\circ} 50^{\prime} 49^{\prime \prime}$ \\
\hline
\end{tabular}

Además de las muestras de agua, se colectaron 12 muestras de sedimentos de la superficie de la laguna considerando los primeros $5 \mathrm{~cm}$. En este caso se delinearon dos transectos periféricos de $2.5 \mathrm{~km}$ de longitud en los márgenes de la laguna, separados entre sí por una distancia promedio de $1.5 \mathrm{~km}$, siendo la separación de cada punto de muestreo en los transectos de aproximadamente $500 \mathrm{~m}$. Los sedimentos (alrededor de $200 \mathrm{~g}$ ) se guardaron en bolsas de polietileno, etiquetaron $\mathrm{y}$ transportaron al laboratorio para su análisis (tabla II, figura 1). 
Tabla II. Coordenadas de toma de muestras de sedimentos.

\begin{tabular}{|l|l|l|}
\hline Clave & Latitud (N) & Longitud (W) \\
\hline S1 & $20^{\circ} 41^{\prime} 27^{\prime \prime}$ & $98^{\circ} 50^{\prime} 19^{\prime \prime}$ \\
\hline S2 & $20^{\circ} 41^{\prime} 17^{\prime \prime}$ & $98^{\circ} 50^{\prime} 20^{\prime \prime}$ \\
\hline S3 & $20^{\circ} 41^{\prime} 05^{\prime \prime}$ & $98^{\circ} 50^{\prime} 32^{\prime \prime}$ \\
\hline S4 & $20^{\circ} 40^{\prime} 55^{\prime \prime}$ & $98^{\circ} 50^{\prime} 45^{\prime \prime}$ \\
\hline S5 & $20^{\circ} 40^{\prime} 48^{\prime \prime}$ & $98^{\circ} 51^{\prime} 02^{\prime \prime}$ \\
\hline S6 & $20^{\circ} 40^{\prime} 51^{\prime \prime}$ & $98^{\circ} 51^{\prime} 27^{\prime \prime}$ \\
\hline S7 & $20^{\circ} 41^{\prime} 16^{\prime \prime}$ & $98^{\circ} 49^{\prime} 41^{\prime \prime}$ \\
\hline S8 & $20^{\circ} 40^{\prime} 54^{\prime \prime}$ & $98^{\circ} 49^{\prime} 47^{\prime \prime}$ \\
\hline S9 & $20^{\circ} 40^{\prime} 39^{\prime \prime}$ & $98^{\circ} 49^{\prime} 52^{\prime \prime}$ \\
\hline S10 & $20^{\circ} 40^{\prime} 25^{\prime \prime}$ & $98^{\circ} 50^{\prime} 02^{\prime \prime}$ \\
\hline S11 & $20^{\circ} 40^{\prime} 16^{\prime \prime}$ & $98^{\circ} 50^{\prime} 17^{\prime \prime}$ \\
\hline S12 & $20^{\circ} 40^{\prime} 03^{\prime \prime}$ & $98^{\circ} 50^{\prime} 34^{\prime \prime}$ \\
\hline
\end{tabular}

Es importante mencionar que, en el mes de muestreo de agua y sedimentos, la laguna ocupaba una superficie aproximada de 10 $\mathrm{Km}^{2}$, correspondiendo a la época de estiaje, con el 60\% de su capacidad máxima. Hacia finales del mismo año, la laguna alcanzó una superficie del orden de $16 \mathrm{Km}^{2}$ después de la época de lluvias. Con la expansión de la llanura de inundación, los transectos muestreados fueron cubiertos en su totalidad.

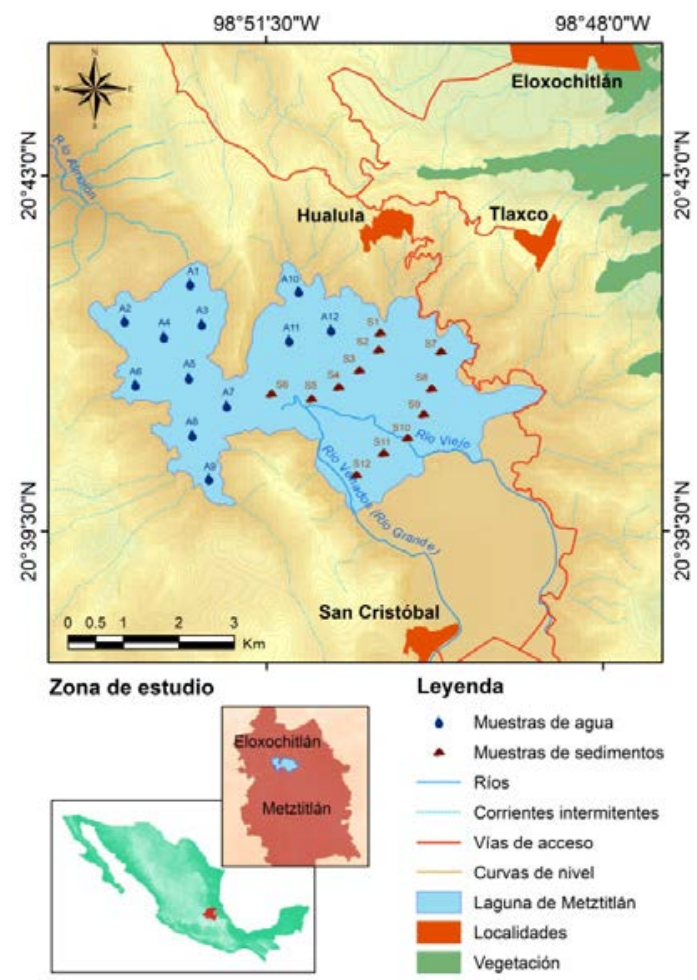

Figura 1. Sitios de muestreo de agua (A) y sedimentos (S) en la laguna de Metztitlán (la laguna se ilustra con su capacidad máxima hacia finales del año 2018).

\subsection{Análisis de agua}

Inicialmente las muestras de agua fueron filtradas empleando filtros de acetato de celulosa con poros de $0.42 \mu \mathrm{m}$. El agua filtrada se separó en dos fracciones, una fracción se conservó con su pH natural y la segunda se aciduló con $\mathrm{HNO}_{3}$ hasta $\mathrm{pH}$ 3.
La fracción de agua no acidulada fue utilizada para determinar el $\mathrm{pH}$ (potenciometría), conductividad eléctrica CE (conductivimetría), sólidos totales disueltos STD (evaporación y sequedad a $105{ }^{\circ} \mathrm{C}$ ), $\mathrm{Cl}-$ (titulación), $\mathrm{CO}_{3}{ }^{2-} \mathrm{y}_{\mathrm{HCO}_{3}}{ }^{-}$ (titulación), $\quad \mathrm{PO}_{4}{ }^{3-}$ (espectrofotometría) y $\mathrm{SO}_{4}{ }^{2-}$ (espectrofotometría) de acuerdo con las técnicas descritas por Sadzawka [10]. Adicionalmente se determinó la concentración de $\mathrm{NO}_{3}{ }^{-}$mediante espectrofotometría empleando la técnica de Rodier [11] y $\mathrm{NH}_{4}^{+}$por espectrofotometría con reactivo de Nessler [12].

La fracción de agua acidulada con $\mathrm{HNO}_{3}$ se utilizó para el análisis elemental por espectrofotometría de emisión atómica en plasma acoplado inductivamente (AES-ICP) en un espectrómetro de emisión óptica Perkin Elmer modelo Óptima 8300, empleando curvas de calibración estándar en el intervalo de concentración de 0 a $25 \mathrm{mg} / \mathrm{L}$ para cada elemento ( $\mathrm{Na}, \mathrm{K}$, $\mathrm{Mg}, \mathrm{Ca}, \mathrm{Si}, \mathrm{y} \mathrm{As})$.

Cada análisis se realizó por triplicado, evaluando su calidad mediante la estimación de la precisión y exactitud, siendo en todos los casos $<5 \%$. La calidad total de los análisis químicos se determinó mediante el error de balance de cargas iónicas \%E, el cual fue $< \pm 5 \%$ [13].

\subsection{Evaluación hidroquímica y salinidad}

Para establecer las características hidroquímicas de las muestras de agua de la laguna de Metztitlán, los datos derivados de los análisis químicos se integraron en los diagramas de van Wirdum [14], Gibbs [15] y Chadha [16], clasificando la dureza de acuerdo con el diagrama de $\mathrm{Li}$ [17] y calculando el índice de saturación (IS) para los principales minerales presentes en el sistema mediante el programa PHREEQC [18].

La salinidad del agua para propósitos de irrigación se evaluó mediante la clasificación USSL [19] empleando la conductividad eléctrica y la relación de adsorción de sodio (RAS), además se utilizó la clasificación de Wilcox [20] que relaciona la conductividad eléctrica con el porcentaje de sodio (PS). Para complementar el diagnóstico se utilizaron los criterios de Lloyd y Heatcothe [21] considerando el carbonato de sodio residual (CSR), así como la clasificación de Donnen [22] utilizando el índice de permeabilidad (IP).

\subsection{Análisis de sedimentos}

Con la finalidad de realizar la caracterización de los sedimentos, previamente se secaron en una estufa a $60{ }^{\circ} \mathrm{C}$ durante 72 h, hasta lograr una masa constante. Posteriormente se determinó su textura empleando el método de Bouyoucos y la clasificación de Shephard [23].

La composición química de las muestras se estableció por fluorescencia de rayos $\mathrm{X}$ en un espectrómetro Rigaku modelo ZSX Primus II (tubo de Rh y ventana de Be). Utilizando perlas de vidrio formadas por fusión de $0.8 \mathrm{~g}$ de muestra y $7.2 \mathrm{~g}$ de fundente $\left(\mathrm{Li}_{2} \mathrm{~B}_{4} \mathrm{O}_{7}\right.$ y $\mathrm{LiBO}_{2}$ en relación 1:1). La fusión se realizó en crisoles de aleación Pt:Au (95:5) empleando LiBr (250 g/l) como agente antiadherente, evaluando las pérdidas por calcinación (PPC) a $950{ }^{\circ} \mathrm{C}$. La concentración de los elementos se determinó mediante el uso de curvas de calibración estándar.

El análisis mineralógico de los sedimentos se realizó mediante difracción de rayos X (método de polvos), empleando un difractómetro marca Bruker, modelo D8 Advance, con radiación $\mathrm{Cu} \mathrm{K} \alpha 1.5406 \AA$. Los patrones de difracción se registraron a temperatura ambiente $\left(18 \pm 2{ }^{\circ} \mathrm{C}\right)$ en el intervalo de $3^{\circ} \leq 2 \theta \leq 80^{\circ}$ empleando un tamaño de paso de $0.020^{\circ}$. Las 
condiciones de operación del tubo de rayos X fueron de $40 \mathrm{kV}$ y $40 \mathrm{~mA}$.

La identificación de las principales fases cristalinas se llevó a cabo mediante el método Hanawalt y el programa Diffract EVA versión 4.1.1. El análisis cuantitativo se realizó mediante la metodología RIR [24] con el programa MATCH! [25].

\section{5 Índices de intemperismo y alteración química}

La definición del grado de alteración de los sedimentos se realizó mediante la estimación del índice de alteración química (CIA), el índice de alteración de plagioclasas (PIA) y el índice de intemperismo químico (CIW), a partir de la composición química de los sedimentos y de las siguientes expresiones [26, 27, 28 y 29]:

$$
\begin{aligned}
& \mathrm{CIA}=\left[\mathrm{Al}_{2} \mathrm{O}_{3} /\left(\mathrm{Al}_{2} \mathrm{O}_{3}+\mathrm{CaO}+\mathrm{Na}_{2} \mathrm{O}+\mathrm{K}_{2} \mathrm{O}\right)\right] \times 100 \\
& \mathrm{PIA}=\left[\left(\mathrm{Al}_{2} \mathrm{O}_{3}-\mathrm{K}_{2} \mathrm{O}\right) /\left(\mathrm{Al}_{2} \mathrm{O}_{3}+\mathrm{CaO}+\mathrm{Na}_{2} \mathrm{O}-\mathrm{K}_{2} \mathrm{O}\right)\right] \times 100 \\
& \mathrm{CIW}=\left[\mathrm{Al}_{2} \mathrm{O}_{3} /\left(\mathrm{Al}_{2} \mathrm{O}_{3}+\mathrm{CaO}+\mathrm{Na}_{2} \mathrm{O}\right)\right] \times 100
\end{aligned}
$$

Donde cada componente se representa en proporciones molares.

\subsection{Factor de enriquecimiento e índice de geoacumulación}

Para evaluar el posible enriquecimiento de los sedimentos con metales traza derivados de actividades antropogénicas se calculó el factor de enriquecimiento (FE) mediante la siguiente expresión [30]:

$$
\mathrm{FE}=[(\mathrm{M} / \mathrm{Fe}) \text { muestra }] /[(\mathrm{M} / \mathrm{Fe}) \text { referencia }]
$$

Donde (M/Fe) muestra, es la proporción entre el metal traza $\mathrm{M}$ $\mathrm{y}$ el elemento normalizador Fe presente en la muestra de sedimento. En tanto que $(\mathrm{M} / \mathrm{Fe})$ referencia, es la proporción entre el metal traza y la concentración de Fe en una referencia geoquímica. Las concentraciones de los elementos para el cálculo del FE se representan en partes por millón.

Una alternativa adicional para establecer la acumulación de metales en sedimentos es mediante el empleo del índice de geoacumulación (IGeo) que se define como [31]:

IGeo $=\log _{2}\left(\mathrm{C}_{\mathrm{n}} / 1.5 \mathrm{~B}_{\mathrm{n}}\right)$

Siendo $\mathrm{C}_{n}$ la concentración del metal $n$ en el sedimento y $\mathrm{B}_{\mathrm{n}}$ el valor de la concentración del metal $n$ en la referencia geoquímica en unidades de partes por millón.

En el presente trabajo de investigación se utilizó como referencia la composición química de una lutita promedio reportada por Turekian y Wedepohl [32] para comparar la concentración de metales traza en los sedimentos respecto de la referencia geoquímica tanto en el cálculo del factor de enriquecimiento (FE) como en el índice de geoacumulación (IGeo).

\section{Resultados y discusión}

\subsection{Características fisicoquímicas del agua de la laguna de Metztitlán}

Los principales parámetros fisicoquímicos de la laguna evaluados en el mes de marzo de 2018 se indican en la tabla III. Destaca la temperatura promedio de $23^{\circ} \mathrm{C}$, con un $\mathrm{pH}$ que varió desde 7.1 hasta 7.5 (casi neutra), siendo la conductividad eléctrica promedio de $531.93 \mu \mathrm{S} / \mathrm{cm}$ y una concentración de sólidos totales disueltos promedio de $530.33 \mathrm{mg} / \mathrm{L}$. La dureza estimada mediante la suma de las concentraciones de $\mathrm{Ca}^{2+} \mathrm{y}$
$\mathrm{Mg}^{2+}$ [33] presentó variaciones desde 172.40 hasta 199.79 $\mathrm{mg} / \mathrm{L}$.

La concentración de cationes mayoritarios disminuye de acuerdo con la serie $\mathrm{Ca}^{2+}>\mathrm{Na}^{+}>\mathrm{Mg}^{2+}>\mathrm{K}^{+}$, en tanto que los aniones mayoritarios disminuyen en el siguiente orden: $\mathrm{HCO}_{3}{ }^{-}$ $>\mathrm{SO}_{4}{ }^{2-}>\mathrm{Cl}^{-}$. Entre los componentes mayoritarios destaca la presencia de sílice con una concentración promedio de 41.23 $\mathrm{mg} / \mathrm{L}$. Otras especies identificadas en las muestras de agua son $\mathrm{NH}_{4}{ }^{+}, \mathrm{NO}_{3}{ }^{-}, \mathrm{PO}_{4}{ }^{3-} \mathrm{y} \mathrm{As}{ }^{3+}$ que se encuentran en concentraciones relativamente bajas. Los valores de la composición química y parámetros físicos de la laguna son característicos de aguas dulces.

Tabla III. Valor mínimo (Min), máximo (Max), promedio (Prom) y desviación estándar (Des) para los parámetros fisicoquímicos determinados en las muestras de agua de la laguna de Metztitlán, Hgo.

\begin{tabular}{|l|l|l|l|l|}
\hline Parámetros & Min & Max & Prom & Des \\
\hline Temp. $\left({ }^{\circ} \mathrm{C}\right)$ & 23 & 23 & 23 & 0 \\
\hline $\mathrm{pH}$ & 7.10 & 7.50 & 7.30 & 0.14 \\
\hline $\mathrm{CE}(\mu \mathrm{S} / \mathrm{cm})$ & 510.00 & 560.00 & 531.93 & 16.19 \\
\hline $\mathrm{STD}(\mathrm{mg} / \mathrm{L})$ & 510.00 & 545.00 & 530.33 & 10.05 \\
\hline Dureza (mg/L) & 172.40 & 199.79 & 183.34 & 7.72 \\
\hline $\begin{array}{l}\text { Componentes } \\
\text { Mayores }\end{array}$ & & & & \\
\hline $\left.\mathrm{Na}^{+} \mathrm{L}\right)$ & 33.60 & 43.60 & 37.55 & 3.25 \\
\hline $\mathrm{K}^{+}$ & 9.22 & 10.80 & 9.95 & 0.48 \\
\hline $\mathrm{Mg}^{2+}$ & 9.90 & 12.80 & 11.43 & 0.74 \\
\hline $\mathrm{Ca}^{2+}$ & 50.90 & 58.90 & 54.56 & 2.37 \\
\hline $\mathrm{SiO}_{2}$ (ac) & 38.00 & 43.30 & 41.23 & 1.41 \\
\hline $\mathrm{HCO}_{3}{ }^{-}$ & 232.20 & 254.70 & 246.52 & 7.82 \\
\hline $\mathrm{Cl}^{-}$ & 25.70 & 32.60 & 28.16 & 2.24 \\
\hline $\mathrm{SO}^{2-}$ & 51.70 & 57.90 & 55.575 & 2.17 \\
\hline $\begin{array}{l}\mathrm{Componentes}^{-} \\
\left.\text {raza }^{+} \mathrm{mg} / \mathrm{L}\right)\end{array}$ & 0.04 & 0.16 & 0.09 & 0.03 \\
\hline $\mathrm{As}^{3+}$ & 0.13 & 0.63 & 0.36 & 0.16 \\
\hline $\mathrm{NH}_{4}{ }^{+}$ & 1.00 & 1.08 & 1.02 & 0.02 \\
\hline $\mathrm{NO}_{3}{ }^{-}$ & 0.77 & 1.18 & 1.03 & 0.13 \\
\hline $\mathrm{PO}_{4}{ }^{3-}$ & -4.76 & -1.61 & -3.68 & 1.04 \\
\hline$\% \mathrm{E}$ & & & \\
\hline
\end{tabular}

\subsection{Hidroquímica y salinidad}

La representación de las concentraciones de $\mathrm{Ca}$ y $\mathrm{Cl}$ respecto de la conductividad eléctrica en el diagrama de van Wirdum ha permitido definir la naturaleza del agua de la laguna como litotrófica (figura 2), es decir, su composición química está regulada por las unidades litológicas que se encuentran en contacto con ella.

De acuerdo con la clasificación de Gibbs, donde se representan los sólidos totales disueltos respecto de las concentraciones de 


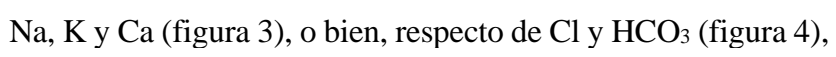
las características químicas del agua son dependientes de la interacción roca-agua, siendo la facie dominante Ca-Mg- $\mathrm{HCO}_{3}$ (figura 5) con una clasificación de agua dulce moderadamente dura (figura 6). De manera general, se ha observado que la composición química y características del agua son homogéneas.

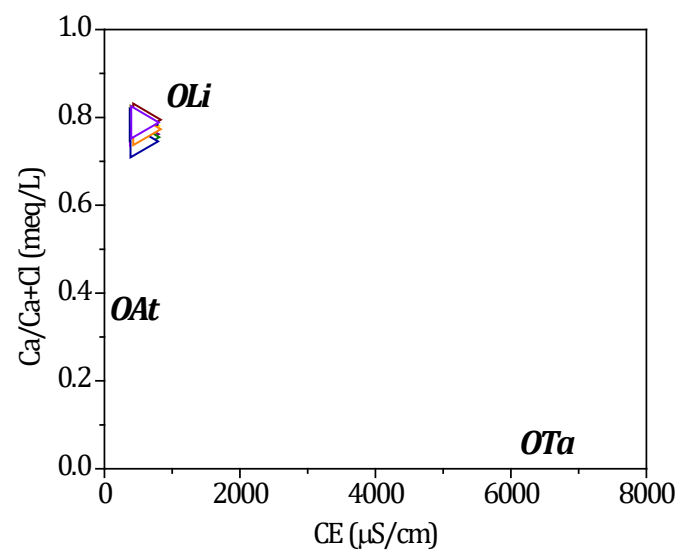

Figura 2. Diagrama de van Wirdum indicando la naturaleza del agua: At atmosférica, Li litotrófica y Ta talasotrófica.

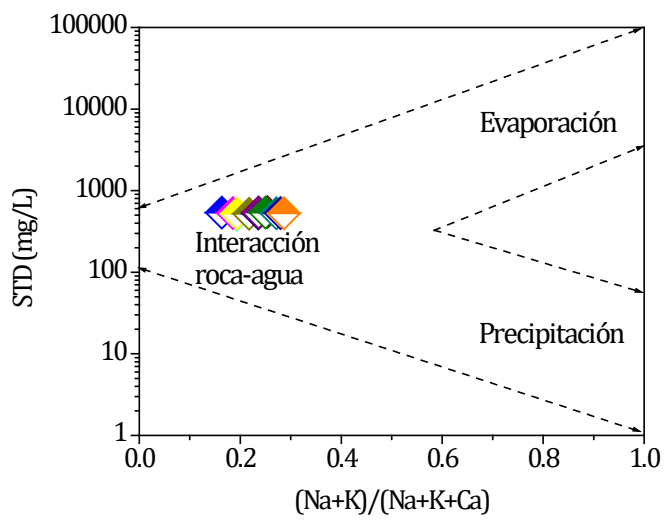

Figura 3. Diagrama de Gibbs con la representación de las concentraciones de sólidos totales disueltos STD y cationes.

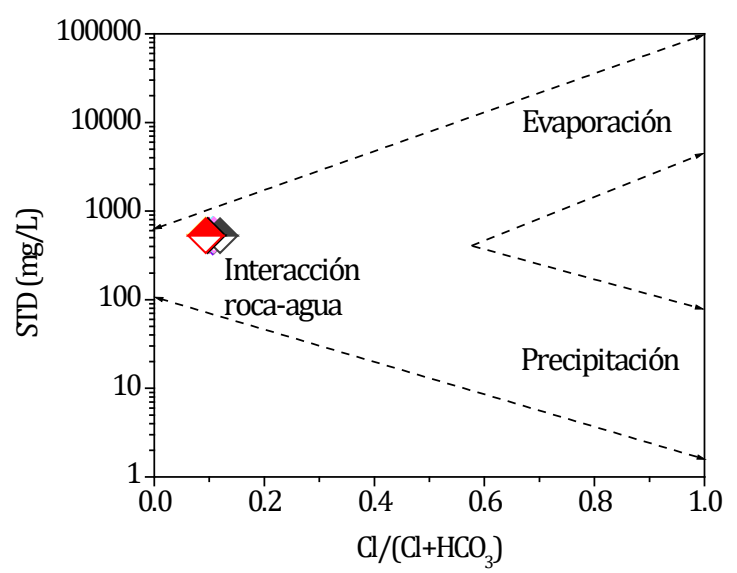

Figura 4. Diagrama de Gibbs representando la concentración de los STD y aniones.

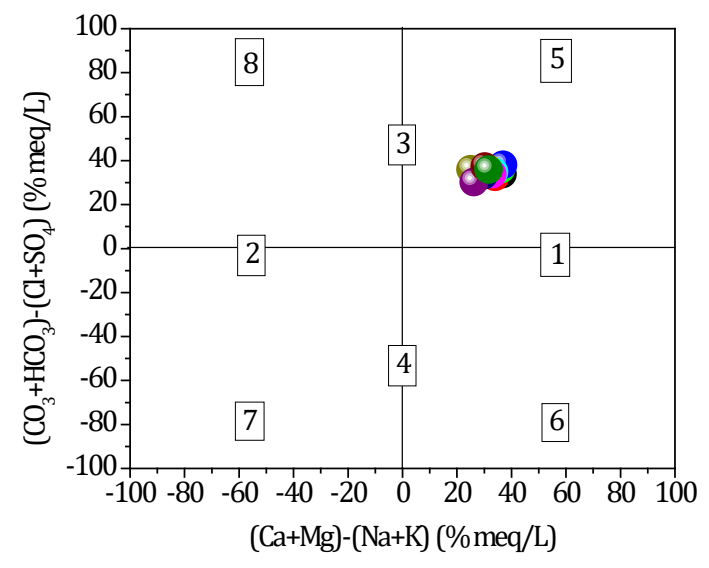

Figura 5. Clasificación de las facies hidroquímicas en el diagrama de Chadha.

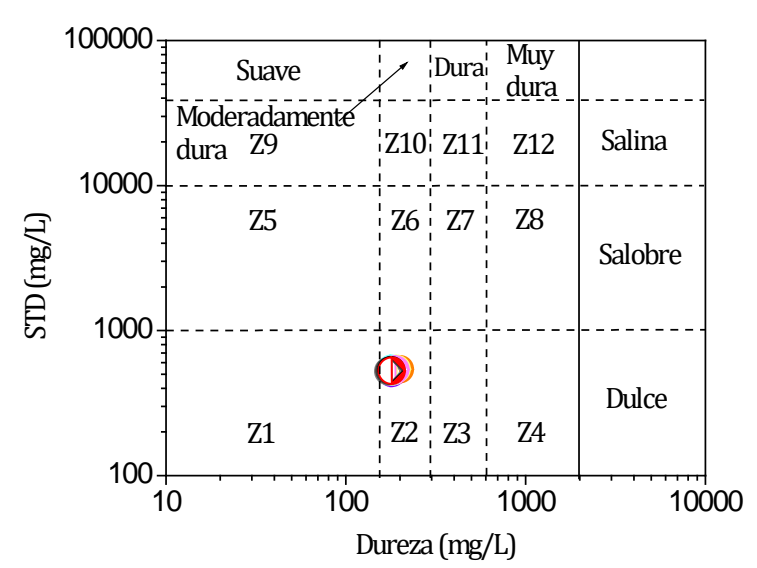

Figura 6. Clasificación de la dureza de las muestras de agua de la laguna.

La evaluación del índice de saturación para un grupo de fases minerales propuestas a partir de la composición química del agua, ha permitido identificar que el sistema se encuentra sobresaturado con cuarzo $\mathrm{SiO}_{2}$ y/o calcedonia $\mathrm{SiO}_{2}$ y se mantiene en la proximidad del equilibrio químico con calcita $\mathrm{CaCO}_{3}$, aragonita $\mathrm{CaCO}_{3}$ y dolomita $\mathrm{CaMg}\left(\mathrm{CO}_{3}\right)_{2}$, manteniéndose insaturado con halita $\mathrm{NaCl}$, yeso $\mathrm{CaSO}_{4} \cdot 2 \mathrm{H}_{2} \mathrm{O}$ y talco $\mathrm{Mg}_{3} \mathrm{Si}_{4} \mathrm{O}_{10}(\mathrm{OH})_{2}$ (figura 7).

Las características químicas del agua de la laguna se pueden explicar considerando que la disolución de minerales como calcita, dolomita y posiblemente aragonita incorporan al sistema iones $\mathrm{Ca}^{2+}$ y $\mathrm{Mg}^{2+}$, así como aniones $\mathrm{CO}_{3}{ }^{2-}$ que en contacto con el agua se transforman en $\mathrm{HCO}_{3}{ }^{-}$resultando una facie $\mathrm{Ca}-\mathrm{Mg}-\mathrm{HCO}_{3}$ que prevalece en el sistema. La presencia de cuarzo y/o calcedonia en el sistema acuático puede ser originado por la disolución de plagioclasas que aportan al sistema sílice hidratada posiblemente como ácido ortosilícico $\mathrm{H}_{4} \mathrm{SiO}_{4}$ que es soluble y sobresatura el agua.

De acuerdo con los valores de la relación de adsorción de sodio (RAS) y la conductividad eléctrica, la salinidad de la laguna de Metztitlán se clasifica como C2S1 (conductividad eléctrica media y bajo contenido en sodio). La relación entre el porcentaje de sodio (PS) y la conductividad eléctrica la clasifica de una calidad permisible a dudosa para su uso como agua de 
riego considerando las categorías de Wilcox. Sin embargo, debido a que los valores del carbonato de sodio residual (CSR) son inferiores a 1.25 se considera que es apta para este propósito, situación que se confirma con los valores del índice de permeabilidad (IP) que se encuentran en el intervalo $25<$ IP $<75$ y corresponden a la clase II: agua de buena calidad para propósitos de irrigación (tabla IV).

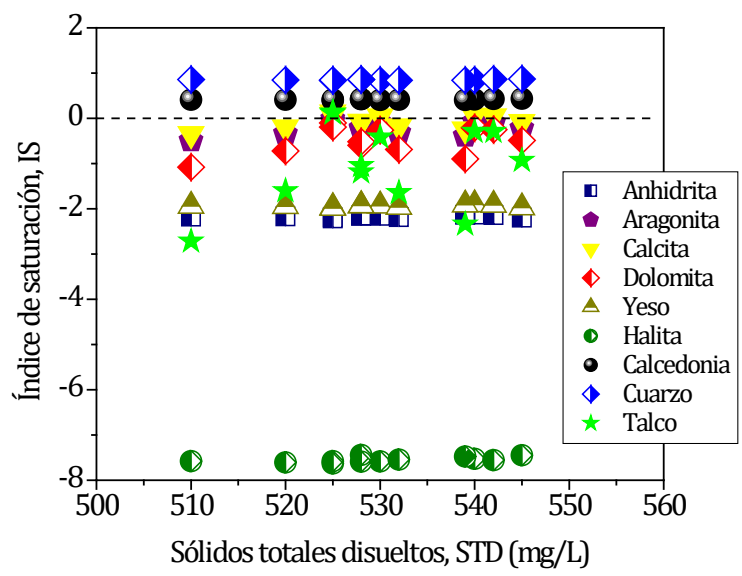

Figura 7. Variación del índice de saturación respecto de los sólidos totales disueltos.

Tabla IV. Índices de salinidad de la laguna de Metztitlán: valores mínimos (Mín), máximos (Máx), promedio (Prom) y desviación estándar (Desv).

\begin{tabular}{|l|l|l|l|l|}
\hline & Min & Max & Prom & Desv \\
\hline RAS & 1.077 & 1.418 & 1.207 & 0.108 \\
\hline PS & 84.108 & 87.671 & 85.578 & 0.947 \\
\hline CSR & 0.161 & 0.600 & 0.381 & 0.132 \\
\hline IP & 42.232 & 46.640 & 44.533 & 1.537 \\
\hline
\end{tabular}

\subsection{Caracterización de los sedimentos}

Los sedimentos de la laguna de Metztitlán corresponden a sedimentos clásticos con un color en seco que varía desde gris claro (10YR 7/2) hasta gris rosáceo (5YR 6/2) [34]. El 75\% de las muestras presenta una textura de limo arenoso, el 16.66\% tiene una textura de limo y el $8.34 \%$ de arena limosa (figura 8). La abundancia relativa de las partículas de arena es de $28.27 \pm$ $10.71 \%$, de limo es de $69.11 \pm 10.97 \%$ y de arcillas es de 2.62 $\pm 1.26 \%$, siendo aproximadamente el $70 \%$ de las partículas inferiores a $50 \mu \mathrm{m}$.

La composición química de los sedimentos muestra una mayor abundancia de $\mathrm{SiO}_{2}$ (con un valor promedio de 42\%) seguida de $\mathrm{CaO}$ (con un promedio de $13.56 \%$ ), $\mathrm{Al}_{2} \mathrm{O}_{3}$ (promedio de $13.49 \%$ ) y $\mathrm{Fe}_{2} \mathrm{O}_{3}$ (promedio de 5.22\%) cuyas concentraciones superan al resto de los óxidos que por lo general son inferiores al 2\% (tabla V).

Los elementos traza identificados se pueden diferenciar por sus características químicas siendo alcalinos (Rb), alcalinotérreos (Sr y Ba), metales de transición (V, Cr, Co, Ni, Cu, Zn, Y, Zr, $\mathrm{Nb}$ y $\mathrm{Pb}$ ) y tierras raras (Th). Los elementos Ba y Sr presentan la mayor concentración en este grupo, seguidos de Zr, Zn y V.
Tabla V. Valores mínimos (Mín), máximos (Máx), promedio (Prom) y desviación estándar (Desv) de la composición química de los sedimentos.

\begin{tabular}{|c|c|c|c|c|}
\hline & Mín & Máx & Prom & Desv \\
\hline $\begin{array}{l}\text { Óxidos } \\
\text { mayores } \\
\text { (\% peso) }\end{array}$ & & & & \\
\hline $\mathrm{SiO}_{2}$ & 31.40 & 52.58 & 42.00 & 6.98 \\
\hline $\mathrm{TiO}_{2}$ & 0.37 & 1.02 & 0.67 & 0.24 \\
\hline $\mathrm{Al}_{2} \mathrm{O}_{3}$ & 8.86 & 18.10 & 13.49 & 3.18 \\
\hline $\mathrm{Fe}_{2} \mathrm{O}_{3}$ & 3.30 & 7.44 & 5.22 & 1.58 \\
\hline $\mathrm{MnO}$ & 0.06 & 0.17 & 0.11 & 0.03 \\
\hline $\mathrm{MgO}$ & 0.97 & 1.57 & 1.11 & 0.15 \\
\hline $\mathrm{CaO}$ & 5.55 & 25.37 & 13.56 & 6.88 \\
\hline $\mathrm{Na}_{2} \mathrm{O}$ & 0.29 & 1.03 & 0.69 & 0.22 \\
\hline $\mathrm{K}_{2} \mathrm{O}$ & 1.19 & 1.84 & 1.54 & 0.20 \\
\hline $\mathrm{P}_{2} \mathrm{O}_{5}$ & 0.14 & 1.74 & 0.35 & 0.44 \\
\hline PPC & 13.30 & 34.13 & 21.06 & 6.03 \\
\hline $\begin{array}{l}\text { Elementos } \\
\text { traza } \\
(\mathrm{mg} / \mathrm{Kg})\end{array}$ & & & & \\
\hline $\mathrm{V}$ & 55 & 303 & 134.50 & 70.62 \\
\hline $\mathrm{Cr}$ & 31 & 60 & 43.91 & 9.72 \\
\hline Co & 6 & 25 & 16.00 & 5.73 \\
\hline $\mathrm{Ni}$ & 19 & 36 & 26.83 & 5.22 \\
\hline $\mathrm{Cu}$ & 17 & 75 & 35.00 & 16.15 \\
\hline $\mathrm{Zn}$ & 57 & 305 & 140.25 & 74.65 \\
\hline $\mathrm{Rb}$ & 49 & 93 & 66.41 & 14.06 \\
\hline $\mathrm{Sr}$ & 138 & 398 & 253.50 & 86.74 \\
\hline $\mathrm{Y}$ & 15 & 41 & 26.08 & 9.19 \\
\hline $\mathrm{Zr}$ & 109 & 290 & 190.91 & 67.69 \\
\hline $\mathrm{Nb}$ & 0 & 14 & 3.83 & 5.49 \\
\hline $\mathrm{Ba}$ & 177 & 975 & 418.83 & 210.42 \\
\hline $\mathrm{Pb}$ & 5 & 48 & 25.50 & 15.94 \\
\hline Th & 3 & 15 & 7.66 & 3.02 \\
\hline
\end{tabular}

La evaluación de los índices de intemperismo y alteración química para los sedimentos, refleja que el $75 \%$ de los sedimentos no presentan intemperismo, mientras que el $25 \%$ restante han sufrido un intemperismo moderado (figuras $9 \mathrm{y}$ 10). La mayoría de las muestras presentan valores de CIA, PIA y CIW inferiores a 50\%, característicos de sedimentos recientes (tabla VI), lo cual es congruente con la naturaleza de las muestras, mismas que son superficiales y que eventualmente entran en contacto con la columna de agua en la laguna. 


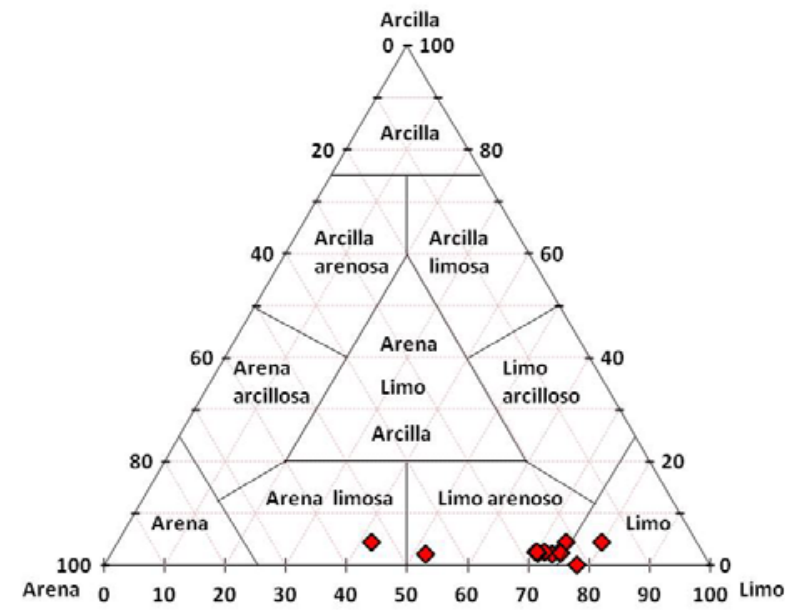

Figura 8. Clasificación textural de los sedimentos.

Tabla VI. Índices de intemperismo de los sedimentos: valores mínimos (Mín), máximos (Máx), promedio (Prom) y desviación estándar (Desv).

\begin{tabular}{|l|l|l|l|l|}
\hline Índice & Mín & Máx & Prom & Desv \\
\hline CIA & 16.5 & 58.5 & 36.88 & 15.77 \\
\hline PIA & 14.5 & 59.3 & 35.85 & 17.06 \\
\hline CIW & 16.81 & 60.27 & 37.72 & 16.46 \\
\hline
\end{tabular}

La mineralogía de los sedimentos determinada mediante difracción de rayos $\mathrm{X}$ permitió identificar cuatro fases cristalinas principalmente: calcita magnésica $\left(\mathrm{Ca}_{0.97} \mathrm{Mg}_{0.3} \mathrm{CO}_{3}\right)$ con numero de archivo JCPDS 89-1304, cuarzo $\left(\mathrm{SiO}_{2}\right)$ con archivo JCPDS 78-1252, plagioclasas como anortita $\left(\mathrm{Ca}_{0.94} \mathrm{Na}_{0.06} \mathrm{Al}_{1.94} \mathrm{Si}_{2.06} \mathrm{O}_{8}\right)$ con archivo JCPDS 84-0751 y caolinita $\left(\mathrm{Al}_{2} \mathrm{Si}_{2} \mathrm{O}_{5}(\mathrm{OH})_{4}\right)$ con archivo JCPDS 14-0164 (figura 11). La abundancia relativa de los minerales en los sedimentos es para cuarzo $33.04 \pm 7.74 \%$, calcita magnésica $26.03 \pm$ $13.02 \%$, plagioclasas $13.81 \pm 3.14 \%$ y caolinita $22.65 \pm 5.63$ $\%$.

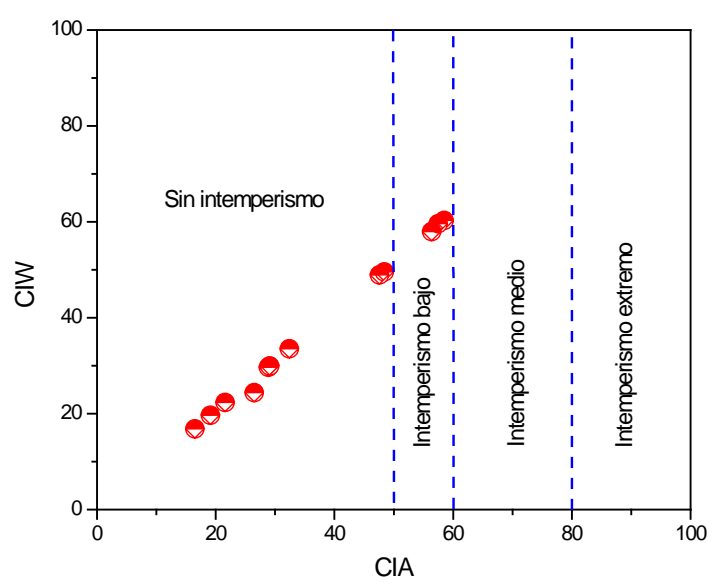

Figura 9. Relación entre los valores de los índices de intemperismo CIW y CIA.

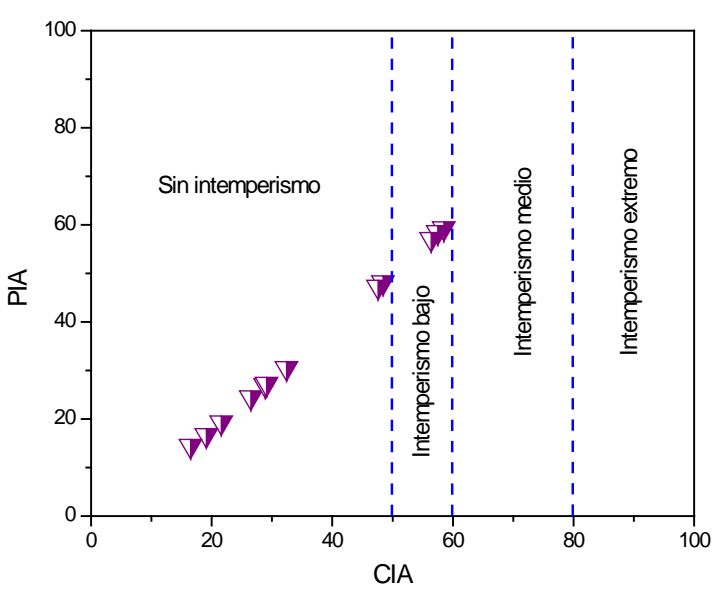

Figura 10. Relación entre los índices de intemperismo PIA y CIA.

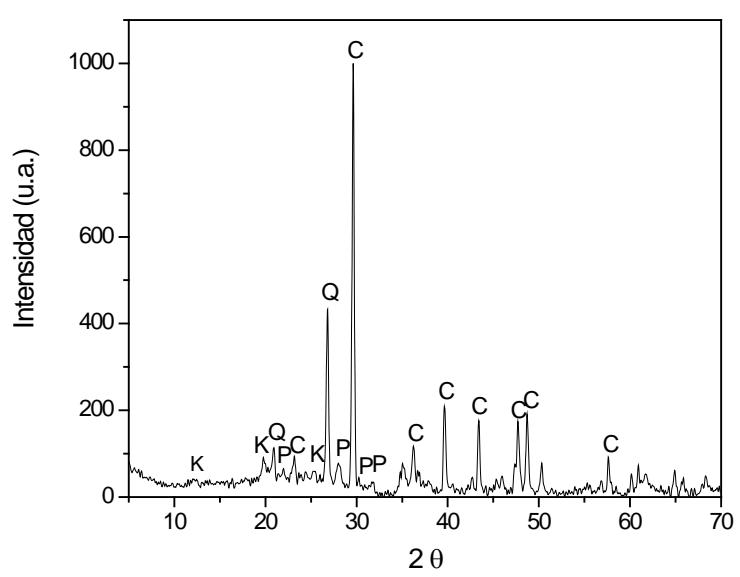

Figura 11. Patrón de difracción de rayos X característico de los sedimentos. Se indican las principales líneas asociadas a calcita magnésica C, cuarzo Q, plagioclasas P y caolinita K.

\subsection{Factor de enriquecimiento y geoacumulación}

La estimación del factor de enriquecimiento (FE) de los sedimentos con los metales $\mathrm{V}, \mathrm{Cr}, \mathrm{Mn}, \mathrm{Co}, \mathrm{Ni}, \mathrm{Cu}, \mathrm{Zn}, \mathrm{Y}, \mathrm{Zr}$, $\mathrm{Nd}, \mathrm{Pb}$ y Th se ha realizado para definir la posible afectación antropogénica de la laguna de Metztitlán considerando las siguientes categorías: $\mathrm{FE}<1$ indica que no hay enriquecimiento; $1<\mathrm{FE}<3$ corresponde a un enriquecimiento menor, $3<\mathrm{FE}<5$ indica un enriquecimiento moderado y $5<\mathrm{FE}<10$ representa un enriquecimiento moderado a severo [35].

En este trabajo se ha empleado al elemento hierro como normalizador y una lutita estándar como referencia geoquímica para comparar las concentraciones de los metales de transición en los sedimentos [32, 36, 37], determinando que el 58.33\% de las muestras presenta un enriquecimiento menor con $\mathrm{V}$, otros elementos que entran en esta categoría son Cr (8.33\%), Mn (75\%), Co (58.33\%), Cu (25\%), Zn (91.66\%), Y (91.66\%), Zr (100\%), Nb (25\%), Pb (66.66\%) y Th (41.66\%). En la categoría de enriquecimiento moderado se encontraron $8.33 \%$ de las muestras con $\mathrm{Mn}$ y $\mathrm{Cu}$, en tanto que se observó enriquecimiento severo con $\mathrm{Zn}$ en el $8.33 \%$ de las muestras de sedimentos (figura 12). 


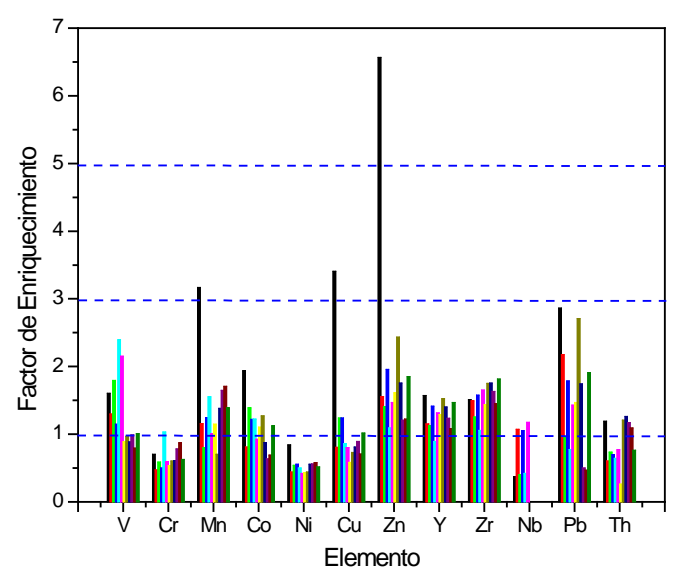

Figura 12. Variación del Factor de Enriquecimiento de metales de transición en los sedimentos de la laguna de Metztitlán.

El índice de geoacumulación IGeo se divide en distintos niveles de contaminación en sedimentos siendo: clase 0 cuando IGeo $<0$ para sedimentos no contaminados; clase I con $0<\mathrm{IGeo}<1$ para sedimentos no contaminados a moderadamente contaminados, clase II con $1<\mathrm{IGeo}<2$ para sedimentos moderadamente contaminados, clase III cuando $2<\mathrm{IGeo}<3$ con sedimentos moderada a fuertemente contaminados, además de otras tres categorías adicionales [31].

En este trabajo se ha empleado una lutita estándar como referencia para definir el índice de geoacumulación de metales de transición en los sedimentos [32, 37], observándose que el $16.66 \%$ se encuentran en la clase I moderadamente contaminados con $\mathrm{V}$, en esta misma categoría se encuentran $\mathrm{Cu}$ (8.33\%), Zn (41.66\%), Y (8.33\%), Zr (25\%) y Pb con 33.33\% de las muestras. En la clase II se encuentran el $8.33 \%$ de los sedimentos moderadamente contaminados con $\mathrm{Zn}$, los elementos restantes corresponden a la clase 0 : no contaminados (figura 13).

Con base en los resultados del Factor de Enriquecimiento y el Índice de Geoacumulación aplicados a los elementos de transición en los sedimentos de la laguna de Metztitlán, se puede distinguir un enriquecimiento relativo y geoacumulación significativa con $\mathrm{V}, \mathrm{Pb}$ y $\mathrm{Zn}$, lo cual implica una posible contaminación por actividades antropogénicas.

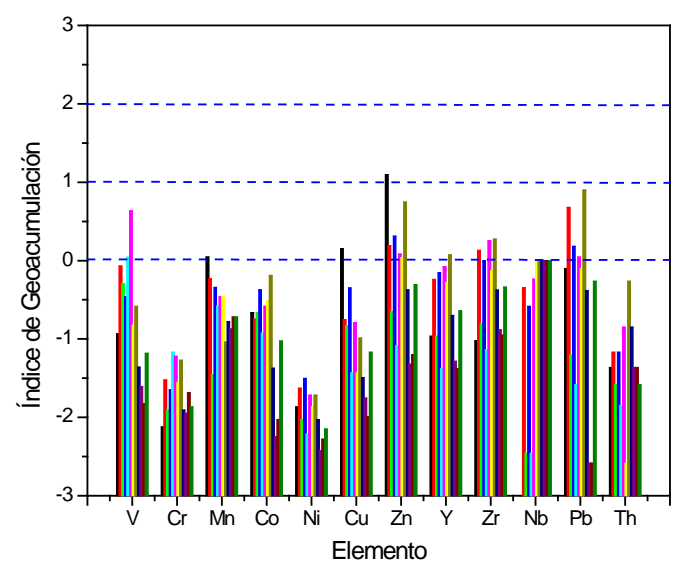

Figura 13. Variación del Índice de Geoacumulación de metales de transición en los sedimentos de la laguna de Metztitlán.

\section{Conclusiones}

Los valores de $\mathrm{pH}$ de la laguna de Metztitlán, próximos a la neutralidad, así como una facie Ca-Mg- $\mathrm{HCO}_{3}$, le confieren una dureza moderada al agua considerada como agua dulce, con una salinidad que la hace apta para propósitos de irrigación, como ha ocurrido desde la época prehispánica.

Las características hidroquímicas de la laguna de Metztitlán influyen notablemente en la migración, desplazamiento, adsorción y precipitación de los metales que se incorporan al sistema acuático a través de las diferentes aportaciones fluviales que convergen en ella, aunado a una constante mezcla de los sedimentos y el agua, denotado por su elevada turbidez.

Debido a que la mayoría de los metales de transición por lo general son insolubles, sobre todo en medios neutros y bicarbonatados, con relativa facilidad pueden ser transformados en carbonatos y precipitarse, acumulándose en los sedimentos.

Es probable que el enriquecimiento y geoacumulación de metales de transición en los sedimentos se presente principalmente por la acumulación de carbonatos metálicos como $\mathrm{ZnCO}_{3}$ y $\mathrm{PbCO}_{3}$, siendo éste el principal mecanismo de depuración del agua. Aunque hay otros procesos que pueden actuar de manera cooperativa como la adsorción y el intercambio iónico, los minerales más importantes para ello en el sistema son la calcita y la caolinita, toda vez que el cuarzo y las plagioclasas poseen una pequeña área superficial y sus propiedades de intercambio iónico y adsorción son limitadas.

El enriquecimiento y geoacumulación relativa de $\mathrm{V}$, Zn y Pb en los sedimentos de la laguna de Metztitlán indican una posible contaminación con estos metales debido a actividades antropogénicas. Sin embargo, es necesario establecer un valor de referencia geoquímica adecuado a este sistema para confirmar los resultados de ésta investigación.

\section{Conflictos de interés}

Los autores declaran no tener ningún conflicto de intereses.

\section{Referencias}

[1] SEMARNAT. NOM-059-SEMARNAT-2010. Protección ambiental. Especies nativas de México de flora y fauna silvestres. Categorías de riesgo y especificaciones para su inclusión, exclusión o cambio. Lista de especies en riesgo [en línea]. Publicada en el Diario Oficial de la Federación el 6 de marzo de 2002. México, Secretaría de Medio Ambiente y Recursos Naturales, 2002.

[2] García-Arizaga MT, Lugo-Hubp J, Palacios D. La obturación de valles por procesos de ladera: el origen de la Vega de Metztitlán (México), in Grandal d'Anglade A, Pagés Valcarlos J, Eds. IV Reunión de Geomorfología, O Castro (A Coruña), España: Sociedad Española de Geomorfología. 1996: 325-335.

[3] Monterrubio CL. El lugar de la luna: Metztitlán en el siglo XVI. 1era Ed. México: UAEH; 2014: 11-14.

[4] Ibáñez A, García J, Pérez A, Álvarez S, Álvarez C, Núñez E. El Lago de Metztitlán, Hidalgo. En De La Lanza G, García J (Comps.). Lagos y Presas de México. México: AGT; 2002: 253-268.

[5] Mendoza M, Quevedo A, Nikolskii I, Rubiños E, Hernández E. Impacto y caudales ambientales del túnel propuesto en la laguna Metztitlán, Hidalgo, México. Tecnología y ciencia del agua. 2011: 2(4); 111-129.

[6] Camargo CT. Inventario de aguas superficiales del Estado de Hidalgo. Pachuca, Hidalgo: Raíces hidalguenses, UAEH. 2000: 40.

[7] Suter M. A neotectonic-geomorphologic investigation of the prehistoric rock avalanche damming Laguna de Metztitlán (Hidalgo State, east-central Mexico). Revista Mexicana de Ciencias Geológicas. 2004: 21(3); 397-411.

[8] Carrasco VBE, Martínez HE, Ramírez AE, Solé VJ. Estratigrafía de la Formación Metztitlán del Plioceno (estado de Hidalgo, Centro-Este de México). Boletín de la Sociedad Geológica Mexicana. 2008: 60 (1); 83-99. 
[9] Comisión Nacional del Agua, CNA. Actualización de la disponibilidad media anual de agua en el acuífero de Metztitlán. Estado de Hidalgo. Gerencia de aguas subterráneas. Subgerencia de Evaluación y Ordenamiento de Acuíferos. 2015: 9-10

[10] Sadzawka RA. Métodos de análisis de aguas para riego. Serie Actas INIA No. 37. Instituto de Investigaciones Agropecuarias. 2006.

[11] Rodier J, Legube B, Merlet N. Análisis del agua. Omega S.A.; 1998: 180.

[12] Welcher FJ (Ed.). Standard methods of chemical analysis. 6th Ed. Vol. 2, Part B. New York: Krieger Publishing Company; 1995: 161-170.

[13] APHA, American Public Health Association. Standard methods for the examination of water and wastewater. 20th Ed. APHA, AWWA, WEF. 1999: 1- 45.

[14] van Wirdum G. Description of water-quality changes in a hydrological cycle for the purpose of nature conservation. In Hooghart JC (Ed.). Water quality in groundwater-flow systems. The Hague, The Netherlands: Commission for Hydrological Research TNO. 1980; 5: 118-143.

[15] Gibbs RJ. Mechanism controlling world water chemistry. Science. 1970; 170 (3962): 1088-1090.

[16] Chadha DK. A proposed new diagram for geochemical classification of natural waters and interpretation of chemical data. Hydrogeol. J. 1999; 7: 431-439.

[17] Li P, Wu J, Qian H. Hydrogeochemistry and quality assessment of shallow groundwater in the southern part of the Yellow River alluvian plain (Zhongwei Section), Northwest China. Earth. Sci. Res. J. 2014; 18 (1): 2738.

[18] Parkhurst DL, Appelo CAJ. User's guide to PHREEQC (ver. 2): A computer program for speciation, batch reaction, one dimensional transport, and inverse geochemical calculations. USGS Water Resources Investigation Report. 1999; 99: 4259.

[19] USSL. Classification of irrigation waters. US Department of Agriculture, Circu 969. Washington. 1954.

[20] Wilcox LV. The quality of water for irrigation use. US Dept. of Agriculture, Tech. Bull., 962, Washington, D.C. 1948: 1-40.

[21] Lloyd JW, Heathcote JA. Natural inorganic hydrochemistry in relation to groundwater: an introduction. 1st Ed. USA: Clarendon; 1985: 294.

[22] Doneen LD. The influence of crop and soil on percolating water. Proc. 1961 Biennial conference on Groundwater Recharge. 1962; 156-163.

[23] Shephard FP. Nomenclature based on sand-silt-clay ratios. J. Sed. Pet. 1954; 24: 151-158.

[24] Langford JI, Louer D. Powder Diffraction. Rep. Prog. Phys. 1996; 59: 131-234.

[25] MATCH!. Phase identification from Powder Diffraction. Crystal Impact.. 2011. V 1.11e.

[26] Fedo CM, Nesbitt HW, Young GM. Unravelling the effects of potassium metasomatism in sedimentary rocks and paleosols, with implications for paleoweathering conditions and provenance. Geology. 1995; 23: 921-924.

[27] Harnois L. The CIW index: a new chemical index of weathering. Sedimentary Geology. 1988; 55: 319-322.

[28] Nesbitt HW, Young GM. Early Proterozoic climates and plate motions inferred from major element chemistry of lutites. Nature. 1982; 299: 715717.

[29] Maynard JB. Chemistry of modern soils as a guide to interpreting Precambrian paleosols. The Journal of Geology. 1992; 100: 279-289.

[30] Hakanson L. An ecological risk index for aquatic pollution control. A sedimentological approach. Water Res. 1980; 14: 975-1001.

[31] Muller G. Die Schwermetallbelsung der sedimente des Neckars und seiner Nebenflusse: Eine Bestandsaufnahme. Chemiker Zeitung. 1981; 105: 156-164.

[32] Turekian KK, Wedepohl KH. Distribution of the elements in some major units of the earth's crust. Geological Society of America. 1961; 72: 175-192.

[33] Weiner ER. Applications of environmental aquatic chemistry: a practical guide. 3rd Ed. País: CRS Press; 2013: 74-94.

[34] Munsell®. Soil color chart. 1994.
[35] Chen CW, Kao CM, Chen CF, Dong CD. Distribution and accumulation of metals in sediments of Kaohiung Harbor, Taiwan. Chemosfere. 2007; 66: 1431-1440.

[36] Amira W, Leghouchi E. Assesment of heavy metal pollution in sediments and their bioaccumulation in Phramites australis from Nil river (Jijel-Algeria), Global NEST Journal. 2018: 20(2); 226-233.

[37] Nowrouzi M, Pourkhabbaz A. Application of geoaccumulation index and enrichment factor for assessing metal contamination in the sediments of Hara Biosphere Reserve, Iran. Chemical Speciation \& Bioavailability. 2014; 26 (2): 99-105. 